\title{
Lagrangians on iterated tangent bundles and the Hamiltonization
}

\author{
Miroslav Kurě̌
}




\title{
LAGRANGIANS ON ITERATED TANGENT BUNDLES AND THE HAMILTONIZATION
}

\author{
MIROSLAV KUREŠ
}

\begin{abstract}
The structure of higher order iterated tangent bundles is studied. Canonical affinors and canonical vector fields on these bundles are geometrically described and differential operators are introduced. Furthermore, possibilities of a generalization of Lagrangian and Hamiltonian formalisms are investigated and a procedure of the Hamiltonization is demonstrated.
\end{abstract}

2000 Mathematics Subject Classification: 58A20, 70G10

Keywords: iterated tangent bundle, nonholonomic jet, Hamiltonization

\section{INTRODUCTION}

Differential geometry provides a good framework for studying Lagrangian and Hamiltonian formalisms of classical mechanics. The approach using semisprays in the place of differential equations brings forth results effectively. Sprays were introduced in the paper of Ambrose et al., [2], for homogeneous second-order differential equations. Grifone's results, [6], make it possible to evaluate a unique EulerLagrange vector field solving fundamental dynamic equations and to construct a connection from a Lagrangian in such a way that the solutions of these equations and the paths of the connection are the same. There are two natural generalizations of the tangent bundle $T M$. The first one is represented by tangent bundles of higher order $T^{r} M$ and corresponds with the classical higher-order calculus of variations. Over the past years, results have been published of de León et al., [3], dealing with semisprays and mutual relations on $T^{r} M$ and motivated by mechanics. The second generalization is represented by higher order iterated tangent bundles. In this paper, we start an investigation into the possibilities of an application of the above approach in this case. First of all, it is necessary to build up a good grounding in a calculus accompanied by a discussion of the structure of iterated tangent bundles. Algebraic properties of investigated higher-order objects are described by Weil algebras as well as by their simplicial structure. The starting points of this interesting view are summarized in the book by White, [19]. The extensive applicability of affinors and generalized Liouville's vector field in differential geometry and mechanics is

The author was supported by GA ČR, grant No. 201/09/0981. 
also known. We construct canonical affinors and vector fields on $\stackrel{\circ}{T}^{r} M$ comparing them with the classification of natural objects on Weil bundles due to Kolár in $[7,10]$. The techniques of finding natural objects are also studied in [9]. Next, we introduce differential operators on a bundle in question. Our definition is a generalization of so-called Tulczyjew's differential operator though our concept is related more significantly to a certain sequence of canonical vector fields. The correspondence between such sequences and ways of differentiating through so-called holonomizing projections is one of the main results of this paper. The new results dealing with the formalisms of mechanics are contained in Section 3. They represent a generalization of the Legendre-Ostrogradskii transformation for the case of iterated tangent bundles. By this, we have introduced Langrangian and Hamiltonian formalism on iterated structures.

\section{ITERATED TANGENT BUNDLES}

\subsection{Preliminaries and motivation}

Let $M, N$ be two smooth manifolds, $\operatorname{dim} M=m, \operatorname{dim} N=n$. As usually, we denote by $J_{a}^{r}(M, N)$ the set of all $r$-jets from $M$ to $N$ with the source $a \in$ $M$. $J_{a}^{r}(M, N)$ is a smooth manifold. The manifold $J_{0}^{1}(\mathbb{R}, M)$ is just the tangent bundle $T M$ on $M$. So, we consider $\pi^{1}: T M \rightarrow M$ the tangent bundle of a smooth $m$-dimensional manifold $M$. Further, the second iterated tangent bundle $T T M=T(T M)$ obtained by the additional application of the functor $T$ possesses the following bundle structures: $\pi^{2}: T T M \rightarrow M, \pi_{1}^{1}:=\pi_{T M}^{1}: T(T M) \rightarrow(T M)$, ${ }_{1} \pi^{1}:=T \pi_{M}^{1}: T(T M) \rightarrow T(M)$. Given some local coordinates $x^{i}$ on $M$, let us denote $x^{i}, y^{i}$ the induced coordinates on $T M$ and $x^{i}, y^{i}, X^{i}, Y^{i}$ the induced coordinates on $T T M$. Then $\pi^{2}:\left(x^{i}, y^{i}, X^{i}, Y^{i}\right) \mapsto\left(x^{i}\right), \pi_{1}^{1}:\left(x^{i}, y^{i}, X^{i}, Y^{i}\right) \mapsto\left(x^{i}, y^{i}\right)$, $1^{1}:\left(x^{i}, y^{i}, X^{i}, Y^{i}\right) \mapsto\left(x^{i}, X^{i}\right)$.

For the mechanical interpretation, we recall Tulczyjew's example of the bundle TTM presented in the monograph of Abraham and Marsden, [1]. The equilibrium configuration of an elastic beam in a Euclidian space with no external forces is a straight line $l$. Small deflections induced by external forces and torques can be represented by points of a plane $M$ perpendicular to the line $l$. The distance measured along $l$ from an arbitrary reference point is denoted by $s$. We select a section of the beam corresponding to an interval $\left[s_{1}, s_{2}\right]$ and assume that the external forces and bending torques are applied to the ends of the section. The configuration manifold of the section of the beam is the product $T M \times T M$ with coordinates $\left(x^{i}, y^{i}, \bar{x}^{i}, \bar{y}^{i}\right)$, $i=1,2$. In the limit $s_{2} \rightarrow s_{1}$, the configuration manifold is the bundle $T T M$ with coordinates $\left(x^{i}, y^{i}, X^{i}, Y^{i}\right)$. 
If we generalize, we obtain the $(r$ th) iterated tangent bundle

$$
\stackrel{\circ}{T}^{r} M=\underbrace{T \ldots T}_{r \text { times }} M=\underbrace{J_{0}^{1}\left(\mathbb{R}, J_{0}^{1}\left(\ldots \left(J_{0}^{1}\right.\right.\right.}_{r \text { times }}(\mathbb{R}, M)) \ldots))
$$

by the multiple application of $J_{0}^{1}$.

\subsection{Iterated tangent bundles and nonholonomic tangent bundles}

The functor $\tilde{J}^{r}$ is defined recurrently as $\tilde{J}^{1}=J^{1}$ and

$$
\tilde{J}^{r}(M, N)=J^{1}\left(M, \tilde{J}^{r-1}(M, N)\right) .
$$

We obtain nonholonomic jets in the sense of Ehresmann, [5]. If we take the subset of nonholonomic jets of $\tilde{J}^{r}(\mathbb{R}, M)$ with the source $0 \in \mathbb{R}$, we have a nonholonomic tangent bundle of order $r \tilde{T}^{r} M=\tilde{J}_{0}^{r}(\mathbb{R}, M)$.

There is a subtle difference between the nonholonomic tangent bundle and the iterated tangent bundle. We can see it through the following definition of the natural equivalence (see Kolár, [8]) $\epsilon_{r}: \tilde{T}^{r} \rightarrow \stackrel{\circ}{T}^{r}$. We define it by induction. For $r=1$, $\epsilon_{1}$ is the identity. Let $t_{u}$ denote the translation on $\mathbb{R}^{k}$ transforming 0 into $u$. If $\sigma_{r-1}: \mathbb{R} \rightarrow \tilde{J}^{r-1}(\mathbb{R}, M)$ is a section, then

$$
\tau_{r-1}(u):=\sigma_{r-1}(u) \circ j_{0}^{r-1}\left(t_{u}\right) \in \tilde{T}^{r-1} M .
$$

Hence, $\epsilon_{r-1}\left(\tau_{r-1}(u)\right) \in \stackrel{\circ}{T}^{r-1} M$ and we define

$$
\epsilon_{r}\left(j_{0}^{1} \sigma_{r}\right):=j_{0}^{1} \epsilon_{r-1}\left(\tau_{r-1}(u)\right) .
$$

Thus, local coordinates of $\stackrel{\circ}{T}^{r} M$ correspond to the local coordinates of $\tilde{T}^{r} M$. For more details about local expressions, see [15].

\subsection{Weil bundles}

We only remark that $\stackrel{\circ}{T}^{r}$ and $\tilde{T}$ are examples of Weil (i. e. product preserving) functors. We refer to [9] for a good introduction to the theory of Weil bundles.

\subsection{Simplicial structure}

For specifications of many general results dealing with Weil bundles, we often need a clear description of them in local coordinates. The use of simplices introduced in White's book, [19], solves this problem adequately just for the case of iterated tangent bundles. We remark that it is also possible to use a more general concept of so-called colored simplices for a wide class of Weil bundles, [13]. A $(r-1)$-simplex is the collection of all non-empty subsets of the set of vertices $\{1, \ldots, r\}$. An $h$-face $\gamma_{h}$ of the simplex, $0 \leq h \leq r-1$, is a subset with $h+1$ elements. We shall designate $\gamma_{h}$ as an ordered $r$-tuple with $a_{p} \in\{0,1\}, p=1, \ldots, r$ and $h+1$ of the elements equals to unity. This corresponds to the subset containing each $s$ such that $a_{s}=1$. The zero tuple is excluded. We write the composition of two disjoint faces $\gamma_{h}, \gamma_{c}$ 
as $\gamma_{h}+\gamma_{c}$. An orientation for a given simplex is an ordering of the vertices. The standard orientation is given by the arrangement $\{1, \ldots, r\}$. Hence we regard such simplices as (standardly) oriented. On the other hand, if we define the simplex as the collection of non-empty subsets of an set of $r$ indistinguishable elements, it is no orientation for it and we call it non-oriented.

White's original procedure of associating simplices with iterated tangent bundles consists in constructing vector spaces of functions from simplices to $\mathbb{R}^{m}$, a definition of a left action of the $r$ th differential group on the fiber product of the frame bundle and this space, etc. It is very exhaustive, but it is a rather long formal process and we do not go into details here. Roughly speaking, it is essential that the local coordinates correspond to faces of simplices in the following way. Given some local coordinates $x^{i}$ on $M$ and $t$ on $\mathbb{R}$, the iterated differentiation of $x^{i}(t)$ determines the induced coordinates $x^{i}, y_{\gamma_{h}}^{i}, h=0, \ldots, r-1$ on $\stackrel{\circ}{T}^{r} M=\underbrace{T \ldots T}_{r \text { times }} M$. In the face $\gamma_{h}$, the elements represent the order of the differentiation. Thus, $a_{s}=1$ means the differentiation $\frac{d}{d t}$ at the $s$ th iteration.

\subsection{Projections}

For every $s, 0 \leq s \leq r$, we denote by $\pi^{s}: \stackrel{\circ}{T}^{s} M \rightarrow M$ the canonical projection to the base. Further, we denote (the notation was first used in [11] for $s=2$ ):

$\pi_{b}^{s}:=\pi_{T^{b}}^{s}: \stackrel{\circ}{T}^{s}\left(\stackrel{o}{T}^{b} M\right) \rightarrow \stackrel{\circ}{T}^{b} M$ the projection with $\stackrel{\circ}{T}^{b} M$ as the base space; such projections are sometimes called direct projections, e.g. in [17]. Iterated tangent bundles together with direct projections represent a so-called full tangential resolution for a manifold $M$.

$a^{\pi^{s}}:=\stackrel{\circ}{T}^{a} \pi^{s}: \stackrel{\circ}{T}^{a}\left(\stackrel{\circ}{T}^{s} M\right) \rightarrow \stackrel{\circ}{T}^{a} M$ the induced projection originating by the posterior application of the functor $\stackrel{\circ}{T}^{a}$; these regular projections are sometimes viewed only as "projections" (the inverted commas e. g. in [17]) or lateral projections (e. g. in [16]).

$a^{\pi}{ }_{b}^{s}:=\stackrel{\circ}{a} \pi_{T^{b} M}^{s}$ the general case containing both previous cases; if $a$ or $b$ equals zero, we do not write them.

Projections $\stackrel{\circ}{T}^{p} M \rightarrow \stackrel{\circ}{T}^{q} M$ are of a type $a_{a} \pi_{b}^{s}$ or they are a composition of projections of such types. The composition $a_{1} \pi_{b_{1}}^{s_{1} \circ} a_{2} \pi_{b_{2}}^{s_{2}}$ is defined only for $a_{1}+b_{1}+s_{1}=$ $a_{2}+b_{2}$ and properties of it are derived in [14]. We note that there are also nonsimplificable compositions of projections of the type $\pi_{a}^{s}$.

\subsection{Structure of subbundles}

For $s=1, \ldots, r$ and $\omega=1, \ldots, s$, let us denote by $T^{\omega} M$ the subset of all $Z \in \stackrel{\circ}{T}^{s} M$ satisfying the $\omega$-condition

$$
\omega-1^{\pi} \pi_{s-\omega}(Z)={ }_{k-1} \pi_{s-k}^{s}(Z)
$$


for all $k, \omega \leq k \leq s$. Of course, $T^{\omega} M$ has a bundle structure (moreover, it is a Weil bundle, because it preserves products) and there are canonical inclusions

$$
\stackrel{1}{T^{s}} M \subset \cdots \subset T^{s} M
$$

and $\stackrel{s}{T} M=\stackrel{\circ}{T} s$. Indeed, if we take $f: N \rightarrow M$, then $\stackrel{\circ}{T}^{s} f: \stackrel{\circ}{T}^{s} N \rightarrow \stackrel{\circ}{T}^{s} M$ and $\stackrel{\circ}{T^{s}} \underset{f}{\left.\stackrel{\omega}{T^{s}} N\right)}=\stackrel{\omega}{T^{s}} M$. In the jet formulation, we easily see, that $\stackrel{1}{T}^{r} M$ coincides with semi-holonomic tangent bundle of order $r$. Furthermore, we can even regard 1

$T^{r} M$ as the holonomic tangent bundle of order $r$ because, in the special case of onedimensional velocities, the difference has only a formal character. For details, see e.g. [14] and [15]. We shall write $\bar{T}^{r} M$ instead $T^{r} M$.

\subsection{Simplices and Weil algebras associated with subbundles}

It is known that $h$-faces, $h=1, \ldots, r-1$, i. e. the coordinates, indices of them have the same number of units, identify in $\bar{T}^{r} M$. Analogously, the $\omega$-condition implies the identification of coordinates with the same number of units in first $r-$ $\omega+1$ indices and with identical rests.

In other words, we have for $T^{r} M, 1<\omega<r$ two subsimplices: the non-oriented $(r-\omega)$-simplex and the oriented $(\omega-2)$-simplex. Faces $\gamma_{h}$ induce faces $\bar{\gamma}_{v}, \gamma_{w}$ in these subsimplices, $v+w+1=h$; we write $\gamma_{h}=\left(\bar{\gamma}_{v}, \gamma_{w}\right)$. As we show, we obtain directly the Weil algebra $\stackrel{\omega}{\mathbb{D}^{r}}$ of the Weil bundle $T^{r} M$. Evaluating the product of two elements and matching the coefficients, we obtain generating equations for the ideal $\mathfrak{i}$ as

$$
\sum_{\bar{\gamma}_{v}} \sum_{\rho \in \mathbb{P}\left(\bar{\gamma}_{v}, 2\right)} t^{\left(\bar{\gamma}_{v_{1}}, \gamma_{w_{1}}\right)} t^{\left(\bar{\gamma}_{v_{2}}, \gamma_{w_{2}}\right)}=\sum_{\bar{\gamma}_{v}} \sum_{\rho \in \mathbb{P}\left(\bar{\gamma}_{v}, 2\right)} t^{\left(\bar{\gamma}_{v}, \gamma_{w}\right)}
$$

for all decompositions of $\gamma_{w}$ to $\gamma_{w_{1}}, \gamma_{w_{2}}$ (where $\mathbb{P}\left(\bar{\gamma}_{v}, 2\right)$ is the set of all decompositions of $v$-face to 2 faces $\bar{\gamma}_{v_{1}}, \bar{\gamma}_{v_{2}}, v_{1}+v_{2}+1=v$, i. e., we sum as to all such decompositions $\left.\rho \in \mathbb{P}\left(\bar{\gamma}_{v}, 2\right)\right)$ and

$$
t^{\left(\bar{\gamma}_{v_{1}}, \gamma_{w_{1}}\right)} t^{\left(\bar{\gamma}_{v_{2}}, \gamma_{w_{2}}\right)}=0
$$

for non-disjoint faces $\gamma_{w_{1}}, \gamma_{w_{2}}$ or for $v_{1}+v_{2}>r-\omega-1$. Then

$$
\stackrel{\omega}{\mathbb{D}^{r}}=\mathbb{R}\left[t^{\gamma_{0}}, \ldots, t^{\gamma_{r-1}}\right] / \mathrm{i} .
$$

\subsection{Holonomizing projections}

The sequence of functors $\stackrel{1}{\rightarrow} T^{s} \subset \cdots \subset \stackrel{s}{\rightarrow} T^{s}$ is respected, be it any choice of projections 
$a_{s-1} \pi_{b_{s-1}}^{1}: \stackrel{\circ}{T}^{s} M \rightarrow \stackrel{\circ}{T}^{s-1} M, \ldots, a_{1} \pi_{b_{1}}^{1}: \stackrel{\circ}{T}^{2} M \rightarrow \stackrel{\circ}{T}^{1} M$ as we see in the following diagram:

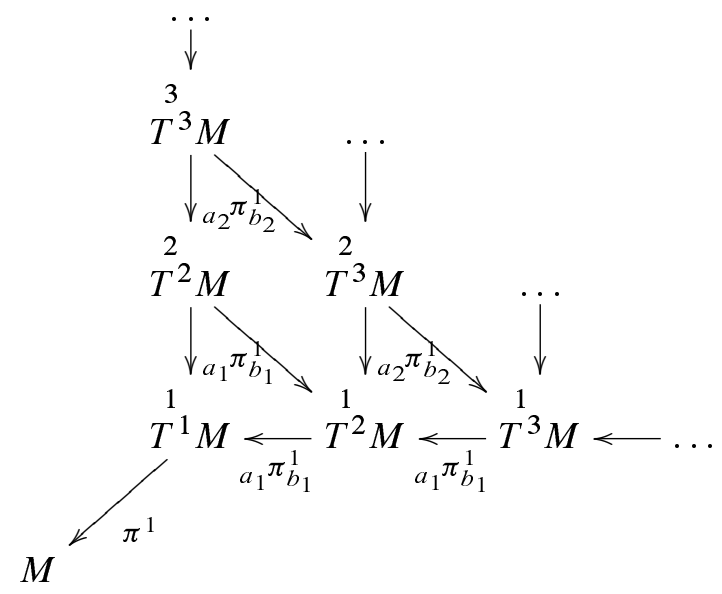

We define the holonomizing projection $\mu: T^{s} M \rightarrow T^{s} M$ (with respect to the projections $\left.{ }_{a_{s-1}} \pi_{b_{s-1}}^{1}: \stackrel{\circ}{T}^{s} M \rightarrow \stackrel{\circ}{T}{ }^{s-1} M, \ldots,{ }_{a_{1}} \pi_{b_{1}}^{1}: \stackrel{\circ}{T}^{2} M \rightarrow \stackrel{\circ}{T}^{1} M\right)$ by

$$
{ }_{a_{1}} \pi_{b_{1}}^{1} \circ \cdots \circ{ }_{a_{s-1}} \pi_{b_{s-1}}^{1} \circ \mu={ }_{a_{1}} \pi_{b_{1}}^{1} \circ \cdots \circ{ }_{a_{s-1}} \pi_{b_{s-1}}^{1}
$$

and it is expressed by the composition of slanting arrows in the diagram.

Altogether, we have $s !$ holonomizing projections $\stackrel{s}{T}^{s} M \rightarrow T^{s} M$.

\subsubsection{Example}

For example, we take $s=3$ and the direct projections $\pi_{2}^{1}, \pi_{1}^{1}$. If we have local coordinates on $\stackrel{\circ}{T}^{3} M$ as $x^{i}, y_{100}^{i}=y^{i}, y_{010}^{i}=X^{i}, y_{110}^{i}=Y^{i}, y_{001}^{i}=\xi^{i}, y_{101}^{i}=$ $\eta^{i}, y_{011}^{i}=\Xi^{i}, y_{111}^{i}=H^{i}$, the coordinate version of the previous diagram is:

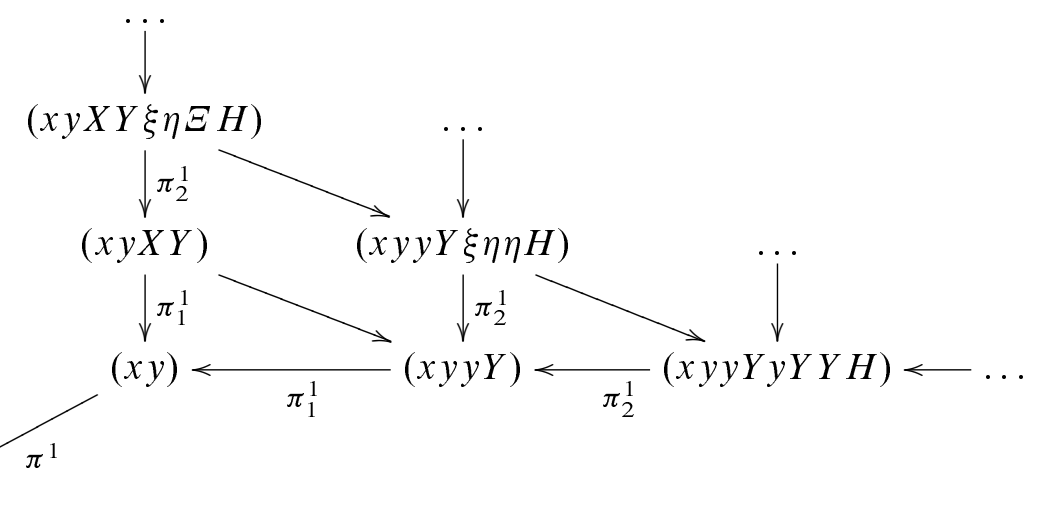




\section{AFFINORS, VECTOR FIELDS AND DIFFERENTIAL OPERATORS}

\subsection{Canonical affinors}

Let $\hat{\pi}^{s}$ denote a family of projections $a_{1} \pi_{b_{1}}^{1}: \stackrel{\circ}{T}^{r} M \rightarrow \stackrel{\circ}{T}^{r-1} M, \ldots, a_{s} \pi_{b_{s}}^{1}: \stackrel{\circ}{T}^{r} M \rightarrow$ $\stackrel{\circ}{T}^{r-1} M, 1 \leq s \leq r, a_{1}+b_{1}=\cdots=a_{s}+b_{s}=r-1$.

Lemma 1. Every family $\hat{\pi}^{s}$ determines a unique projection $\stackrel{\circ}{T}^{r} M \rightarrow \stackrel{\circ}{T}^{r-s} M$.

Proof. The proof is easy from the simplicial point of view. The projections $\stackrel{\circ}{T}^{r} M \rightarrow$ $\stackrel{\circ}{T}^{r-1} M$ mean projections of $(r-1)$-simplex onto $(r-2)$-faces. If we have $s$ projections $\stackrel{\circ}{T}^{r} M \rightarrow \stackrel{\circ}{T}^{r-1} M, s<r$, an $(r-s-1)$-face or a projection $\stackrel{\circ}{T}^{r} M \rightarrow \stackrel{\circ}{T}^{r-s} M$ is determined. If $s=r$, we have the projection $\pi^{r}: \stackrel{\circ}{T}^{r} M \rightarrow M$.

For every such a family $\hat{\pi}^{s}$, we have the canonical monomorphism

$$
\text { i : } V^{\hat{\pi}^{s}} \stackrel{\circ}{T} M \longrightarrow T \stackrel{\circ}{T} M
$$

and the canonical epimorphism

$$
\mathbf{s}: T \stackrel{\circ}{T}^{r} M \longrightarrow T \stackrel{\circ}{T}^{r-s} M \times \stackrel{\circ}{T} r-s_{M} \stackrel{\circ}{T}^{r} M
$$

of vector bundles over $\stackrel{\circ}{T}^{r} M$, where $V^{\hat{\pi}^{s}}$ means the verticality with respect to all projections belonging to the family $\hat{\pi}^{s}$ and the fibration $\stackrel{\circ}{T}^{r} M \rightarrow \stackrel{\circ}{T}^{r-s} M$ is through the determined projection from the previous lemma. There is also the canonical isomorphism (in fact the vertical lift)

$$
\mathbf{h}: T \stackrel{\circ}{T}^{r-s} M \times_{\stackrel{\circ}{T}^{r-s} M} \stackrel{\circ}{T}^{r} M \longrightarrow V^{\hat{\pi}^{s}} \tilde{T}^{r} M .
$$

Since, by an affinor $A$ on a manifold $Y$, we mean a $(1,1)$-tensor field, which we can identify with a linear morphism $A: T Y \rightarrow T Y$ over id $_{M}$, the canonical affinor is defined as

$$
A:=\mathbf{i} \circ \mathbf{h} \circ \mathbf{s} .
$$

For fixed $r, s$ we obtain $\left(\begin{array}{l}r \\ s\end{array}\right)$ affinors in this way. Particularly, for $s=1$ we obtain $r$ affinors. We shall call them the primary affinors. We obtain all generators of natural affinors (except the identical affinor) in this way, where by a natural affinor on a natural bundle $F$ over $m$-dimensional manifolds we mean a system of $(1,1)-$ tensor fields $A_{M}: T F M \rightarrow T F M$ for every $m$-dimensional manifold $M$ satisfying $T F f \circ A_{M}=A_{N} \circ T F f$ for every local diffeomorphism $f: M \rightarrow N$. All natural affinors on iterated tangent bundles are described in [12], where it is proved:

Proposition 1. All natural affinors on $\stackrel{\circ}{T}^{r} M$ constitute a $2^{r}$-parameter family linearly generated by the identical affinor $A_{0}$ and by affinors $A_{\gamma_{c}}$, where $\gamma_{c}$ represent 
all c-faces, $0 \leq c \leq r-1$, which work in this way:

$$
\begin{aligned}
d y_{\gamma_{c}}^{i} & =d x^{i} \\
d y_{\gamma_{c}+\gamma_{h}}^{i} & =d y_{\gamma_{h}}^{i}, \quad h \leq r-c-2 .
\end{aligned}
$$

Indeed, we have $2^{r}$ canonical affinors on $\stackrel{\circ}{T}^{r} M$ including the identical affinor. It is essential that the classification of all natural objects makes assurance double sure that our list of canonical affinors is complete. Evidently,

$$
A_{\gamma_{h}} \circ A_{\gamma_{c}}=A_{\gamma_{h}+\gamma_{c}}
$$

for disjoint faces $\gamma_{h}, \gamma_{c}$, elsewhere the composition vanishes. It follows that it is possible to generate all canonical affinors by the iterations of primary affinors.

\subsection{Canonical vector fields}

Now we construct canonical vector fields on $\stackrel{\circ}{T}^{r} M$. For a projection ${ }_{a}{ }_{b}^{1}: \stackrel{\circ}{T}^{r} M \rightarrow$ $\stackrel{\circ}{T}^{r-1} M$, let $\alpha$ denote the canonical section of the vector bundle $T \stackrel{\circ}{T}^{r-1} M \times{ }_{T^{r-1} M}$ $\stackrel{\circ}{T}^{r} M$. The canonical vector field is defined as

$$
\zeta:=\mathbf{i} \circ \mathbf{h} \circ \alpha .
$$

We shall call such vector fields the primary vector fields. For the construction of subsequent canonical vector fields, we apply canonical affinors to primary vector fields.

We remark that a natural vector field on a natural bundle $F$ over $m$-dimensional manifolds is a system of vector fields $\zeta_{M}: F M \rightarrow T F M$ for every $m$-dimensional manifold $M$ satisfying $T F f \circ \zeta_{M}=\zeta_{N} \circ T F f$ for every local diffeomorphism $f: M \rightarrow N$. The natural vector fields on $F$ can be interpreted as so-called absolute natural operators $C^{\infty} T M \rightarrow C^{\infty} T F M$ transforming vector fields on $M$ into vector fields on $F M$. For more details about natural objects see [9]. Let us add that we have entailed the geometrical interpretation of generators of natural vector fields by our construction. Now, we take the sequence $\zeta_{1}, \ldots \zeta_{r}$ of canonical vector fields with these properties:

(i) $\zeta_{1}$ is a primary vector field;

(ii) $\zeta_{s+1}$ is obtained from $\zeta_{s}$ by applying a primary affinor (for every $s=1, \ldots, r-$ 1).

Then we call this sequence the normal sequence of canonical vector fields. By some combinatorics, we see that we have constructed $r 2^{r-1}$ canonical vector fields, which we can order to $r$ ! normal sequences. We need the following assertion.

Proposition 2. Every normal sequence of canonical vector fields $\zeta_{1}, \ldots \zeta_{r}$ on $\stackrel{\circ}{T}^{r} M$ determines a unique holonomizing projection $\mu: \stackrel{\circ}{T}^{r} M \rightarrow \bar{T}^{r} M$. 
Proof. The normal sequence of canonical vector fields $\zeta_{1}, \ldots \zeta_{r}$ is given by a primary vector field $\zeta_{1}$ and by primary affinors $A_{2}, \ldots, A_{r}$, where $\zeta_{s}=A_{s}\left(\zeta_{s-1}\right)$ for $s=2, \ldots, r$. Every such affinor has a form $A_{\gamma_{0}}$. We know that, for non-disjoint faces $\gamma_{h}, \gamma_{c}$, the composition of affinors $A_{\gamma_{h}} \circ A_{\gamma_{c}}$ vanishes. It follows that affinors $A_{2}, \ldots, A_{r}$ are different and are related to $r-1$ different projections $\stackrel{\circ}{T}^{r} M \rightarrow$ $\stackrel{\circ}{T}^{r-1} M$. Further, the vector field $\zeta_{1}$ is also related to a projection $\stackrel{\circ}{T}^{r} M \rightarrow \stackrel{\circ}{T}^{r-1} M$. Of course, this projection is different from all those mentioned above: if not, the application of such an affinor gives the zero vector field. So, we have $r$ different projections $\stackrel{\circ}{T}^{r} M \rightarrow \stackrel{\circ}{T}^{r-1} M$. Hence, we have families of projections $\hat{\pi}^{s}, s=1, \ldots, r$, which we construct gradually by adding projections related to $A_{2}, \ldots, A_{r}$ to the projection related to $\zeta_{1}$. In Lemma 1, it was proved that every family $\hat{\pi}^{s}$ determines a unique projection $\stackrel{\circ}{T}^{r} M \rightarrow \stackrel{\circ}{T}{ }^{r-s} M$. We obtain projections

$$
\stackrel{\circ}{T}^{r} M \rightarrow \stackrel{\circ}{T}^{r-1} M, \stackrel{\circ}{T}^{r} M \rightarrow \stackrel{\circ}{T}^{r-2} M, \ldots, \stackrel{\circ}{T}^{r} M \rightarrow \stackrel{\circ}{T}^{1} M, \stackrel{\circ}{T}^{r} M \rightarrow M
$$

in this way. They determine uniquely projections

$$
\stackrel{\circ}{T}^{r} M \rightarrow \stackrel{\circ}{T}^{r-1} M, \stackrel{\circ}{T}^{r-1} M \rightarrow \stackrel{\circ}{T}^{r-2} M, \ldots, \stackrel{\circ}{T}^{2} M \rightarrow \stackrel{\circ}{T}^{1} M, \stackrel{\circ}{T}^{1} M \rightarrow M .
$$

We fix the choice of projections $a_{r-1} \pi_{b_{r-1}}^{1}: \stackrel{\circ}{T}^{r} M \rightarrow \stackrel{\circ}{T}^{r-1} M, \ldots, a_{1} \pi_{b_{1}}^{1}: \stackrel{\circ}{T}^{2} M \rightarrow$ $\stackrel{\circ}{T}^{1} M$ (the projection $\stackrel{\circ}{T}^{1} M \rightarrow M$ is unique trivially). So, the holonomizing projection $\mu: \stackrel{\circ}{T}^{r} M \rightarrow \bar{T}^{r} M$ is determined, cf. Section 1.8.

Remark 1 . Conversely, every holonomizing projection determines a unique normal sequence of canonical vector fields.

\subsection{Differential operators}

We introduce the following differential operator $d_{T}$ on $\stackrel{\circ}{T}^{r} M$ related to a given normal sequence of canonical vector fields $\zeta_{1}, \ldots \zeta_{r}$. Let $\mu$ be the holonomizing projection with respect to bundle projections $a_{s} \pi_{b_{s}}^{1}: \stackrel{\circ}{T}^{s+1} M \rightarrow \stackrel{\circ}{T}^{s} M, s=1, \ldots, r-$ 1 derived from $\zeta_{1}, \ldots \zeta_{r}$ (Proposition 2). Let $\psi_{r}: \stackrel{r}{r}^{r} M \rightarrow T\left(\stackrel{\circ}{T}^{r-1} M\right)$, be the mapping given by $\mu\left(\tau_{r}\right) \mapsto j_{0}^{1} \tau_{r-1}$. (See Sections 1.2 and 1.8 for the definitions of $\tau_{r}$ and $\mu$.) $\psi_{r}$ can be viewed as a generalization of the map $\phi_{r}$ using by Tulczyjew in [18]. Then, locally,

$$
\psi_{r}\left(x^{i}, y_{\bar{\gamma}_{h}}^{i}\right)=y_{\bar{\gamma}_{0}}^{i} \frac{\partial}{\partial x^{i}}+y_{\bar{\gamma}_{1}}^{i} \sum_{\gamma_{0}} \frac{\partial}{\partial y_{\gamma_{0}}^{i}}+\cdots+y_{\bar{\gamma}_{r}}^{i} \sum_{\gamma_{r-1}} \frac{\partial}{\partial y_{\gamma_{r-1}}^{i}},
$$

where $\bar{\gamma}_{0}, \ldots, \bar{\gamma}_{r-1}$ are the faces belonging to $\bar{T}^{r} M=\mu\left(\stackrel{\circ}{T}^{r} M\right)$ and $\bar{\gamma}_{r}$ is the face with nothing but units. We use this map to construct the differential operator $d_{T}$, which maps each function $F$ on $\stackrel{\circ}{T}^{r} M$ into a function $d_{T} F$ on $\stackrel{\circ}{T}^{r+1} M$, defined by

$$
d_{T} F\left(\tau_{r+1}\right)=d F\left(\tau_{r}\right)\left(\psi_{r} \circ \mu\left(\tau_{r+1}\right)\right) .
$$


Since $d_{T}(F G)=\left(d_{T} F\right) G+F\left(d_{T} G\right), d_{T}$ extends to $p$-forms on $\stackrel{\circ}{T}^{r} M$ in such a way that $d_{T} d=d d_{T}$. Further, $d_{T}^{s}:=\underbrace{d_{T} \circ d_{T}}_{r \text { times }}$.

Remark 2. As we have $r$ ! holonomizing projections related to $r$ ! normal sequences of canonical vector fields, we have also $r$ ! operators $d_{T}$, in other words $r$ ! ways of differentiating. Nevertheless, a hard and fast rule of using them is respecting the described correspondence. On purpose, we desist from more complicated denotation with an indexing of $d_{T}$ purposely.

\section{LEGENDRE-OSTROGRADSKII TRANSFORMATION}

After preceding preparation, we can now formulate the foundations of formalisms of mechanics on $\stackrel{\circ}{T}^{r} M$. Let us consider a Lagrangian $\mathscr{L}: \stackrel{\circ}{T}^{r} M \rightarrow \mathbb{R}$. For vector fields $\zeta_{s}: \stackrel{\circ}{T}^{r} M \rightarrow T \stackrel{\circ}{T}{ }^{r} M, s=1, \ldots, r$ from a normal sequence of canonical vector fields, we have induced vector fields $\bar{\zeta}_{s}: \bar{T}^{r} M \rightarrow T \bar{T}^{r} M$ such that $\bar{\zeta}_{s} \circ \mu=T \mu \circ \zeta_{s}$. We define the energy as

$$
E:=\sum_{\zeta_{1}, \ldots, \zeta_{r}} \sum_{s=1}^{r} \frac{(-1)^{s-1}}{(r-s) ! s !} d_{T}^{s-1}\left(\bar{\zeta}_{s} \mathscr{L}\right)-\mathscr{L}
$$

where $\sum_{\zeta_{1}, \ldots, \zeta_{r}}$ denotes the sum over all normal sequences of vector fields.

Proposition 3. The energy is a function on $V^{\hat{\pi}^{2 r-2}} \ldots V^{\hat{\pi}^{r}} \stackrel{\circ}{T} M$.

Proof. $\mathscr{L}$ is a function on $\stackrel{\circ}{T}^{r} M$ and $\bar{\zeta}_{s} \mathscr{L}$ is also a function on $\stackrel{\circ}{T}^{r} M$ for an arbitrary $s=1, \ldots, r$. We transfer to the bundle ${ }^{\circ}{ }^{r+1} M$ by the application of $d_{T}$. Since we have

$$
d_{T} F=y_{\bar{\gamma}_{0}}^{i} \frac{\partial F}{\partial x^{i}}+y_{\bar{\gamma}_{1}}^{i} \sum_{\gamma_{0}} \frac{\partial F}{\partial y_{\gamma_{0}}^{i}}+\cdots+y_{\bar{\gamma}_{r+1}}^{i} \sum_{\gamma_{r}} \frac{\partial F}{\partial y_{\gamma_{r}}^{i}}
$$

for a arbitrary function $F: \stackrel{\circ}{T}^{r} M \rightarrow \mathbb{R}$, we have $y_{\bar{\gamma}_{0}}^{i}, \ldots, y_{\bar{\gamma}_{r}}^{i}$ as coordinates on $\stackrel{\circ}{T}^{r} M$ and, in addition, we have only new coordinates $y_{\bar{\gamma}_{r+1}}^{i}$, which are on $\stackrel{\circ}{T}^{r+1} M$. So, we are on a vertical bundle, more precisely on the bundle, which is vertical with respect to all $r$ projections $\stackrel{\circ}{T}^{r} M \rightarrow \stackrel{\circ}{T}^{r-1} M$. This verticality we have denoted $V^{\hat{\pi}^{r}}$. Analogously, we obtain the verticality $V^{\hat{\pi}^{r+1}}$ by the application of $d_{T}^{2}=d_{T} \circ d_{T}$, etc. Finally, for $d_{T}^{r-1}$ we are really on the bundle $V^{\hat{\pi}^{2 r-2}} \ldots V^{\hat{\pi}^{r}} \stackrel{\circ}{T} r M$.

For every normal sequence of canonical vector fields we define the partial momenta $\wp_{i}^{\bar{\gamma}_{h}}, h=0, \ldots, r-1$ as

$$
\wp_{i}^{\bar{\gamma}_{h}}:=\sum_{c=0}^{r-h-1} \frac{(-1)^{c}}{(r-h-c-1) !(h+c+1) !} d_{T}^{c} \frac{\partial \mathscr{L}}{\partial y_{\bar{\gamma}_{h+c}}^{i}} .
$$


If we evaluate all partial momenta for all normal sequences of canonical vector fields, it is evident that we obtain partial momenta, indices of them are the same faces. We define the (total) momenta $p_{i}^{\gamma_{h}}, h=0, \ldots, r-1$ as the sum of the partial momenta with equal indices, i.e. $p_{i}^{\bar{\gamma}_{h}}:=\sum \wp_{i}^{\bar{\gamma}_{h}}$.

Paper [19] studies a so-called bundle of boundaries $B^{r-1} M$. In the simplicial language, we obtain it by removing the maximal face from the associated $(r-1)$ simplex. Geometrically,

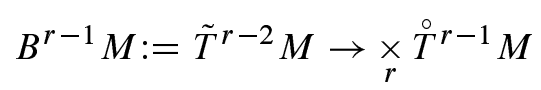

where $\tilde{T}^{r-2} M \rightarrow \underset{r}{\times}$ denotes the fiber (Whitney) product over $\stackrel{\circ}{T}^{r-2} M$ with respect to $r$ different projections $\stackrel{\circ}{T}^{r} M \rightarrow \stackrel{\circ}{T}^{r-1} M$. It is known that $\stackrel{\circ}{T}^{r} M \rightarrow B^{r-1} M$ is the affine bundle. We discover easily:

Proposition 4. The partial momenta represent local coordinates on $T^{*} \bar{T}^{r-1} M$. The momenta represent local coordinates on $T^{*} B^{r-1} M$.

Proof. We recall that the classical way of defining the cotangent bundle is the union of vector spaces over the reals, which are composed of differentials at a point of a given manifold. So, the first part of the assertion follows immediately from the definition of the partial momenta because the indices of which are the faces belonging to $\bar{T}^{r} M=\mu\left(\stackrel{\circ}{T}^{r} M\right)$. We see the second part equally clearly; it is sufficient to recall the geometrical definition of $B^{r-1} M$.

The natural generalization of the Legendre transformation to higher-order theories is called the Legendre-Ostrogradskii transformation and it is well-known for the bundle $T^{r} M$; it enables the Hamiltonization of mechanical problems (see, e. g., [4]). If we take the two preceding assertions into this consideration, we have immediately the main theoretical result:

Corollary 1. If $\mathscr{L}$ is a regular Lagrangian, then the introduced energy and momenta enable the generalized Legendre-Ostrogradskii transformation

$$
\widetilde{\mathrm{Leg}}: V^{\hat{\pi}^{2 r-2}} \ldots V^{\hat{\pi}^{r}} \stackrel{\circ}{T}{ }^{r} M \rightarrow T^{*} B^{r-1} M,
$$

which is a natural generalization of the Legendre-Ostrogradskii transformation Leg: $T^{2 r-1} M \rightarrow T^{*} T^{r-1} M$.

For the completion, we state the following essential formula.

Proposition 5. The local coordinate expression of the energy is

$$
E=\sum_{h=0}^{r-1} p_{i}^{\gamma_{h}} y_{\gamma_{h}}^{i}-\mathscr{L} .
$$


Proof. The equalities

$$
\begin{aligned}
E & =\sum_{\zeta_{1}, \ldots, \zeta_{r}} \sum_{s=1}^{r} \frac{(-1)^{s-1}}{(r-s) ! s !} d_{T}^{s-1}\left(\bar{\zeta}_{s} \mathscr{L}\right)-\mathscr{L} \\
& =\sum_{\zeta_{1}, \ldots, \zeta_{r}} \sum_{s=1}^{r} \frac{(-1)^{s-1}}{(r-s) ! s !} d_{T}^{s-1}\left(\sum_{h=0}^{r-s} y_{\bar{\gamma}_{h}}^{i} \frac{\partial \mathscr{L}}{\partial y_{\bar{\gamma}_{h+s-1}}^{i}}\right)-\mathscr{L} \\
& =\sum_{\zeta_{1}, \ldots, \zeta_{r}} \sum_{h=0}^{r-1} \sum_{c=0}^{r-h-1} \frac{(-1)^{c}}{(r-c-1) !(c+1) !} d_{T}^{c}\left(y_{\bar{\gamma}_{h}}^{i} \frac{\partial \mathscr{L}}{\partial y_{\bar{\gamma}_{h+c}}^{i}}\right)-\mathscr{L} \\
& =\sum_{\zeta_{1}, \ldots, \zeta_{r}} \sum_{h=0}^{r-1} y_{\bar{\gamma}_{h}}^{i} \sum_{c=0}^{r-h-1} \frac{(-1)^{c}}{(r-h-c-1) !(h+c+1) !} d_{T}^{c}\left(\frac{\partial \mathscr{L}}{\partial y_{\bar{\gamma}_{h+c}}^{i}}\right)-\mathscr{L} \\
& =\sum_{\zeta_{1}, \ldots, \zeta_{r}} \sum_{h=0}^{r-1} y_{\bar{\gamma}_{h}}^{i} \wp_{i}^{\bar{\gamma}_{h}}-\mathscr{L}=\sum_{h=0}^{r-1} p_{i}^{\gamma_{h}} y_{\gamma_{h}}^{i}-\mathscr{L}
\end{aligned}
$$

lead one immediately to the formula given above.

Example. We take $\stackrel{\circ}{T}^{3} M$ and $m=1$ with local coordinates $x, y_{100}=y, y_{010}=$ $X, y_{110}=Y, y_{001}=\xi, y_{101}=\eta, y_{011}=\Xi, y_{111}=H$. Given is also the Lagrangian $\mathscr{L}=(2 y+X) \eta+H^{2}$.

We have 12 canonical vector fields in 6 normal sequences:

$$
\begin{aligned}
& \zeta_{1}=y \frac{\partial}{\partial y}+Y \frac{\partial}{\partial Y}+\eta \frac{\partial}{\partial \eta}+H \frac{\partial}{\partial H}, \quad \zeta_{2}=y \frac{\partial}{\partial \eta}+Y \frac{\partial}{\partial H}, \quad \zeta_{3}=y \frac{\partial}{\partial H}, \\
& \zeta_{1}=y \frac{\partial}{\partial y}+Y \frac{\partial}{\partial Y}+\eta \frac{\partial}{\partial \eta}+H \frac{\partial}{\partial H}, \quad \zeta_{2}=y \frac{\partial}{\partial Y}+\eta \frac{\partial}{\partial H}, \quad \zeta_{3}=y \frac{\partial}{\partial H}, \\
& \zeta_{1}=X \frac{\partial}{\partial X}+Y \frac{\partial}{\partial Y}+\Xi \frac{\partial}{\partial \Xi}+H \frac{\partial}{\partial H}, \quad \zeta_{2}=X \frac{\partial}{\partial \Xi}+Y \frac{\partial}{\partial H}, \quad \zeta_{3}=X \frac{\partial}{\partial H}, \\
& \zeta_{1}=X \frac{\partial}{\partial X}+Y \frac{\partial}{\partial Y}+\Xi \frac{\partial}{\partial \Xi}+H \frac{\partial}{\partial H}, \quad \zeta_{2}=X \frac{\partial}{\partial Y}+\Xi \frac{\partial}{\partial H}, \quad \zeta_{3}=X \frac{\partial}{\partial H}, \\
& \zeta_{1}=\xi \frac{\partial}{\partial \xi}+\eta \frac{\partial}{\partial \eta}+\Xi \frac{\partial}{\partial \Xi}+H \frac{\partial}{\partial H}, \quad \zeta_{2}=\xi \frac{\partial}{\partial \Xi}+\eta \frac{\partial}{\partial H}, \quad \zeta_{3}=\xi \frac{\partial}{\partial H}, \\
& \zeta_{1}=\xi \frac{\partial}{\partial \xi}+\eta \frac{\partial}{\partial \eta}+\Xi \frac{\partial}{\partial \Xi}+H \frac{\partial}{\partial H}, \quad \zeta_{2}=\xi \frac{\partial}{\partial \eta}+\Xi \frac{\partial}{\partial H}, \quad \zeta_{3}=\xi \frac{\partial}{\partial H} .
\end{aligned}
$$


Every sequence determines a holonomizing projection $\mu$ and it provides us with $d_{T}$ and with $\bar{\zeta}_{s}, s=1,2,3$. We have

$$
\bar{\zeta}_{1}=y \frac{\partial}{\partial y}+Y \frac{\partial}{\partial Y}+Y \frac{\partial}{\partial Y}+H \frac{\partial}{\partial H}, \quad \bar{\zeta}_{2}=y \frac{\partial}{\partial Y}+Y \frac{\partial}{\partial H}, \quad \bar{\zeta}_{3}=y \frac{\partial}{\partial H}
$$

and we evaluate

$$
\sum_{s=1}^{3} \frac{(-1)^{s-1}}{(3-s) ! s !} d_{T}^{s-1}\left(\bar{\zeta}_{s} \mathscr{L}\right)=y\left(\eta+\frac{1}{3} \ddot{H}\right)+Y\left(-\frac{1}{3} \dot{H}\right)+H\left(\frac{1}{3} H\right) .
$$

$\dot{H}, \ddot{H}$ are new coordinates on $\stackrel{\circ}{T}^{4} M, \stackrel{\circ}{T}^{5} M$, respectively. The expressions in the brackets are the partial momenta. Analogously, we obtain

$$
\begin{gathered}
y\left(-\frac{1}{2} \eta+\frac{1}{3} \ddot{H}\right)+\eta\left(y+\frac{1}{2} X-\frac{1}{3} \dot{H}\right)+H\left(\frac{1}{3} H\right), \\
X\left(\frac{1}{2} \eta+\frac{1}{3} \ddot{H}\right)+Y\left(-\frac{1}{3} \dot{H}\right)+H\left(\frac{1}{3} H\right) \\
X\left(\frac{1}{2} \eta+\frac{1}{3} \ddot{H}\right)+\Xi\left(-\frac{1}{3} \dot{H}\right)+H\left(\frac{1}{3} H\right), \\
\xi\left(\frac{1}{3} \ddot{H}\right)+\Xi\left(-\frac{1}{3} \dot{H}\right)+H\left(\frac{1}{3} H\right) \\
\xi\left(-\frac{3}{2} \eta+\frac{1}{3} \ddot{H}\right)+\eta\left(y+\frac{1}{2} X-\frac{1}{3} \dot{H}\right)+H\left(\frac{1}{3} H\right) .
\end{gathered}
$$

We sum the corresponding partial momenta to obtain the energy as

$$
\begin{aligned}
E=y\left(\frac{1}{2} \eta\right. & \left.+\frac{2}{3} \ddot{H}\right)+X\left(\eta+\frac{2}{3} \ddot{H}\right)+\xi\left(-\frac{3}{2} \eta+\frac{2}{3} \ddot{H}\right)+Y\left(-\frac{2}{3} \dot{H}\right) \\
& +\eta\left(2 y+X-\frac{2}{3} \dot{H}\right)+\Xi\left(-\frac{2}{3} \dot{H}\right)+H(2 H)-(2 y+X) \eta-H^{2} .
\end{aligned}
$$

The expressions in the brackets are the momenta $p_{100}=p, p_{010}=q, p_{001}=$ $r, p_{110}=P, p_{101}=Q, p_{011}=R, p_{111}=\Pi$. We evaluate the Hamiltonian $\mathscr{H}=y p+X q+\xi r+Y P+\eta Q+\Xi R+\frac{1}{4} \Pi^{2}-(2 y+X) \eta$.

Finally, let us consider the standard holonomic case. If we choose a holonomizing projection $\mu$, e.g. $\mu:(x y X Y \xi \eta \Xi H) \mapsto(x y y Y y Y Y H)$, we have the Lagrangian $\mu(\mathscr{L})=3 y Y+H^{2}$. We can evaluate energy $\left(2 y \ddot{H}-2 Y \dot{H}+H^{2}\right)$ and Hamiltonian $\left(y p+Y P+\frac{1}{4} \Pi^{2}-3 y Y\right)$ in the classical way and verify that they represent the holonomized $E$ and $\mathscr{H}$, respectively.

\section{REFERENCES}

[1] R. Abraham and J. E. Marsden, Foundations of Mechanics. New York: Benjamin / Cummings, 1978.

[2] W. Ambrose, R. S. Palais, and I. M. Singer, "Sprays," Anais. Acad. Brasileria Ciencia, vol. 32, pp. 163-178, 1960. 
[3] L. C. de Andrés, M. de León, and P. R. Rodrigues, "Connections on tangent bundles of higher order associated to regular lagrangians," Geom. Dedic., vol. 39, pp. 17-28, 1991.

[4] M. de León and P. R. Rodrigues, " ${ }^{k}$ almost-tangent structures and the hamiltonization of higherorder field theories," J. Math. Phys., vol. 30, pp. 1351-1353, 1996.

[5] C. Ehresmann, "Extension du calcul des jets aut jets non holonomes," C.R.A.S. Paris, vol. 239, pp. 1762-1764, 1954.

[6] J. Grifone, "Structure presque-tangente et connexions," Annales de l'institut Fourier, vol. 22.1, pp. 287-334, 1972.

[7] I. Koláŕ, "On the natural operators on vector fields," Ann. Glob. Anal. Geom., vol. 6, pp. 109-117, 1988.

[8] I. Kolár. "Bundle functors of the jet type," in Differential Geometry and Applications. Brno: Masaryk University, 1999, pp. 231-237.

[9] I. Kolář, P. W. Michor, and J. Slovák, Natural Operations in Differential Geometry. Springer Verlag, 1993.

[10] I. Kolář and M. Modugno, "Torsions of connections on some natural bundles," Diff. Geom. and its Appl., vol. 2, pp. 1-16, 1992.

[11] M. Kureš, "Some properties of connections on iterated tangent bundles," Arch. Mech., vol. 50, pp. 459-466, 1998.

[12] M. Kureš, "Affinors and connections in higher order geometry," in Differential Geometry and Applications, 1999, pp. 239-245.

[13] M. Kureš, "On the simplicial structure of some weil bundles," Supplem. ai Rendic. del Circ. Matem. di Palermo, vol. 63, pp. 131-140, 2000.

[14] M. Kureš, "Weil algebras of generalized higher order velocities bundles," Cont. Math., vol. 288, pp. 358-362, 2001.

[15] M. Kureš, "Looking at osculating bundles through the semiholonomity equalizations," Adv. and Appl. in Math. Sci., vol. 10, no. 7, pp. 1-8, 2011.

[16] E. Pavlíková, "Lateral projections of non-holonomic jets," Math. Slovaka, vol. 23, no. 2, pp. 184190, 1973.

[17] R. G. Pond, "Lift order problems for ordinary differential equations on manifolds," Int. Jour. of Theor. Phys., vol. 36, pp. 715-741, 1997.

[18] W. Tulczyjew, "Sur la différentiel de lagrange," C.R.A.S. Paris, vol. 280, pp. 1295-1298, 1975.

[19] J. E. White, The Method of Iterated Tangents with Applications in Local Riemannian Geometry. London: Pitman, 1982.

Author's address

\section{Miroslav Kureš}

Institute of Mathematics, Brno University of Technology, Technická 2, 61669 Brno, Czech Republic

E-mail address: kures@efme.vutbr.cz 